\title{
Emergency centre investigation of first-onset seizures in adults in the Western Cape, South Africa
}

\author{
A B Smith, MB ChB; D J van Hoving, MB ChB, DipPEC, MMed, MScMedSci; L A Wallis, MB ChB, FCEM, MD \\ Division of Emergency Medicine, Faculty of Medicine and Health Sciences, Stellenbosch University, South Africa
}

Corresponding author: D J van Hoving (nvhoving@sun.ac.za)

Background. Patients with first-onset seizures commonly present to emergency centres (ECs). The differential diagnosis is broad, potentially life-threatening conditions need to be excluded, and these patients need to be correctly diagnosed and appropriately referred. There are currently no data on adults presenting with first-onset seizures to ECs in South Africa.

Objective. To review which investigations were performed on adults presenting with first-onset seizures to six ECs in the Western Cape Province.

Methods. A prospective, cross-sectional study was conducted from 1 July 2011 to 31 December 2011. All adults with first-onset seizures were included; children and trauma patients were excluded. Subgroup analyses were conducted regarding HIV status and inter-facility variation. Results. A total of 309 patients were included. Computed tomography (CT) scans were planned in 218 (70.6\%) patients, but only performed in 169; 96 (56.8\%) showed abnormalities judged to be causative (infarction, intracerebral haemorrhage and atrophy being the most common). At least $80 \%$ of patients $(n=247)$ received a full renal and electrolyte screen, blood glucose testing and a full haematological screen. Lumbar puncture (LP) was performed in 67 (21.7\%) patients, with normal cerebrospinal fluid findings in 51 (76.1\%). Only 27 (8\%) patients had an electroencephalogram, of which 5 (18\%) were abnormal. There was a statistically significant difference in the number of CT scans $(p=0.002)$ and LPs $(p<0.001)$ performed in the HIV-positive group $(n=49)$.

Conclusion. This study demonstrated inconsistency and wide local variance for all types of investigations done. It emphasises the need for a local guideline to direct doctors to appropriate investigations, ensuring better quality patient care and potential cost-saving.

S Afr Med J 2013;103(10):723-727. DOI:10.7196/SAMJ.6821 


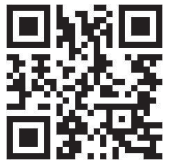

Seizures are a common presentation to emergency centres (ECs). The worldwide incidence of acute symptomatic seizures is 29 - 39 per 10000 per year. ${ }^{[1]}$

The differential diagnosis of a patient presenting with first-onset seizures is broad, and emergency physicians should take a careful history, conduct a thorough examination and perform appropriate investigations, with a view not only to excluding potentially life-threatening conditions, but also to achieve a correct diagnosis and determine appropriate management and referral (disposal) of patients. ${ }^{[2]}$

High-quality guidelines regarding the investigation of adults with first-onset seizures are available in the UK and the USA, but similar South African (SA) guidelines are non-existent. ${ }^{[2,3]}$ Local data regarding the incidence or types of seizures were also lacking. International guidelines suggest that the following investigations are appropriate: neuroimaging, electroencephalogram (EEG) and laboratory investigations. ${ }^{[2,3]}$

Neuroimaging can be achieved by either computed tomography (CT) scanning or magnetic resonance imaging (MRI) to exclude causes such as tumours, haemorrhage and infarction. MRI is diagnostically superior to CT scan for detecting certain intracranial lesions, but is not readily available. ${ }^{[4]}$ Literature shows a broad range of abnormal CT scan findings (ranging from $12 \%$ to $41 \%$ ) in patients presenting with seizures. This yield increases to $59 \%-82 \%$ when the patient has an abnormal neurological examination. ${ }^{[4,5]}$ Because a CT scan is useful to exclude catastrophic conditions in patients with first-onset seizures, guidelines advise a CT scan if resources do not permit MRI. ${ }^{[2,3]}$

There is little evidence supporting routine widespread biochemical investigations. Themostcommonbiochemicalabnormalitiesassociated with seizures are hypo- or hyperglycaemia and hyponatremia ${ }^{[1]}$ and these should be tested for in all cases. ${ }^{[5,7]}$ Some abnormal findings (e.g. raised white cell count or hyperglycaemia) may be a direct result of the seizure activity. ${ }^{[6]}$ Other laboratory investigations should be ordered as clinical circumstances dictate. ${ }^{[5,6]}$ Unless contraindicated, a lumbar puncture (LP) should be performed whenever an intracranial infection is suspected, in all immunocompromised patients, and in young patients where no obvious cause for a seizure is identified. ${ }^{[8-10]}$

An EEG is considered a useful investigation to diagnose epilepsy and has an average abnormal yield of $29 \% .^{[3,1]}$ While a normal EEG does not exclude epilepsy, it may predict seizure recurrence. ${ }^{[4]}$ EEGs are usually only recorded at specialist units, and together with a high false negative rate, some experts believe that it is an unnecessary EC investigation. ${ }^{[12]}$

HIV/AIDS patients frequently present with seizures ${ }^{[13,14]}$ Physicians must adopt a low threshold for neuroimaging and an LP is indicated if judged to be a safe procedure. ${ }^{[9,13]}$ SA has a high HIV infection rate $(10.5 \%),{ }^{[15]}$ but there are little data on seizures in the SA HIV-positive population. $^{[14,15]}$

\section{Objective}

As there are currently no data regarding this group of patients, nor how they are investigated, the aim of this study was to review which special investigations are being undertaken on adults with first-onset seizures in Western Cape ECs.

\section{Methods \\ Study design \\ A prospective, cross-sectional study design was used.}

\section{Study setting and population}

Six hospitals situated within the Western Cape Province were selected by convenience sampling - two tertiary centres and four smaller hospitals (at secondary and district level). The two tertiary level hospitals, Groote
Schuur Hospital (GSH) and Tygerberg Hospital (TBH) are both large referral centres with 24-hour access to CT and MRI scans and specialist radiologists. The four secondary and district level hospitals have limited specialist services and limited access to special investigations. Three secondary hospitals (New Somerset, G F Jooste and Paarl hospitals) have on-site CT scanners that are operational during office hours. Victoria Hospital (VH) does not have an on-site CT scanner and refer their patients to GSH for this investigation. Together, these hospitals serve a population of approximately 3.4 million, many of whom are underprivileged or unemployed. ${ }^{[15]}$

All adults who presented to the ECs with first-onset seizures during the study period (1 July 2011 to 31 December 2011) were eligible for inclusion. First-onset seizures were defined as the first episode of a focal or generalised seizure in a patient not known to have epilepsy or any other seizure disorder. ${ }^{[1]}$ Focal seizures were defined as complex focal seizures or simple focal seizures without secondary generalisation. Generalised seizures were defined as primary generalised tonic-clonic seizures, and focal seizures with secondary tonic-clonic generalisation.

Paediatric patients (under the age of 13 years) were excluded owing to the high incidence of febrile seizures. Also, SA protocols exist for children with first-onset seizures. Patients with post-traumatic seizures (presenting within 1 week after head injury) were also excluded as their work-up differs from that of non-trauma patients.

\section{Measurements}

Healthcare personnel working in the relevant ECs were asked to help to identify eligible patients. The principal investigator also reviewed the EC registers weekly, to minimise missed cases. (All ECs have an EC register where the patients' details, clinical problem and disposal are recorded).

Data were extracted from patients' folders and entered into an electronic datasheet (Microsoft Excel) by the principal investigator. Variables collected included basic demographic data (age and sex), type of seizure, HIV status and investigations performed. Patients who were scheduled for investigations as outpatients outside of the 6-month study period were followed up for a further 6 weeks to ensure that results of their investigations were captured.

\section{Data analysis}

Statistical analyses were performed by the Centre for Statistical Services at Stellenbosch University. Simple descriptive statistics were used to describe the data. The association between two nominal variables was investigated with contingency tables and likelihood ratio $\chi^{2}$ tests, specifically Pearson's $\chi^{2}$ test for independence. Subgroup analyses were conducted regarding HIV status and inter-facility variation. A significance level of $p \leq 0.05$ was used.

\section{Results}

A total of 1241 patients were identified during the study period. Nine hundred and thirty-two patients were excluded (not first time seizures $(n=892)$, missing folders $(n=25)$, paediatric patients $(n=10)$, trauma related seizures $(n=5))$. Thus, 309 patients were included in the final analysis. The study population's demographic details, seizure types and HIV status are presented in Table 1. GSH evaluated the majority of patients $(n=84 ; 27.2 \%)$ (Table 2$)$.

CT scans formed part of the investigation of 218 (70.6\%) patients, $151(69.3 \%)$ on an emergent basis and 67 (30.7\%) electively. Only 18 (26.8\%) of the elective CT scans were eventually performed. Ninetysix $(56.8 \%)$ of the CT scans performed were abnormal and interpreted as revealing a cause for the seizure (Fig. 1); the remainder $(n=73$; 43.2\%) were normal or showed an abnormality that was judged not 
Table 1. Demographics, seizure type and HIV status of patients presenting with first-onset seizures

$n(\%)$

\begin{tabular}{ll}
\hline & $\boldsymbol{n}(\%)$ \\
\hline Age (years) & $36(11.7)$ \\
$13-20$ & $54(17.5)$ \\
$21-30$ & $69(22.3)$ \\
$31-40$ & $63(20.4)$ \\
$41-50$ & $44(14.2)$ \\
$51-60$ & $27(8.7)$ \\
$61-70$ & $16(5.2)$ \\
$>70$ & \\
Gender & $120(38.8)$ \\
Male & $189(61.2)$ \\
Female & \\
HIV status & $49(15.8)$ \\
Positive & $53(17.2)$ \\
Negative & $207(67)$ \\
Unknown & \\
Seizure type & $41(13.3)$ \\
Focal & $268(86.7)$ \\
Generalised &
\end{tabular}

Table 2. Distribution of patients with first-onset seizures across hospitals

\begin{tabular}{ll}
\hline Hospital & $\boldsymbol{n}(\%)$ \\
\hline Groote Schuur & $84(27.2)$ \\
Victoria & $56(18.1)$ \\
Tygerberg & $51(16.5)$ \\
New Somerset & $51(16.5)$ \\
G F Jooste & $34(11)$ \\
Paarl & $33(10.7)$ \\
Total, $N$ & $309(100)$
\end{tabular}

to be causative of the seizure. All 18 elective scans were normal. No patient received an MRI as part of their work-up.

A total of 1762 different blood investigations were performed (Fig. 2), the most common being serum glucose ( $n=282 ; 91.2 \%)$. More than $80 \%$ of patients had renal function tests (urea and creatinine), with sodium and potassium being the most common electrolytes tested. Toxicology screening was performed on 11 (3.6\%) patients. Sixty-seven (21.7\%) patients had an LP, with 51 (76.1\%) showing normal results. HIV testing was performed on only $5(1.6 \%)$ patients.

Twenty-seven patients (8\%) underwent EEGs, 19 (6.2\%) on an elective basis. Five (18.5\%) EEGs, all urgently performed, showed one or more abnormalities.

\section{HIV status}

The number of patients with a confirmed HIV-positive status was almost equal to those known to be HIV-negative (Table 1). A statistically significant difference existed between the HIV-positive group and the other two groups regarding the number of CT scans $(p=0.002)$ and the number of LPs $(p<0.001)$ performed. Table 3 summarises this subgroup analysis (excluding blood investigations).

\section{Inter-facility variability}

Two-thirds ( $n=100 ; 66.2 \%)$ of the urgent CT scans were performed at the tertiary level hospitals. The majority of elective CT scans $(n=58$; $86.6 \%$ ) were arranged from secondary level hospitals. There was a statistically significant difference between secondary and tertiary level hospitals regarding the number of CT scans ordered $(p<0.001)$. Fourteen (73.7\%) of all elective EEGs were from one hospital (VH). All eight urgent EEGs were from the two tertiary hospitals. The biochemical tests performed also varied between facilities; however, because these represented very small numbers of patients, statistical significance was not tested. Thyroid function tests were performed on $16(5.2 \%)$ patients, the majority at TBH $(n=7)$ and $\mathrm{VH}(n=7)$. Serological testing for syphilis was performed on $22(7.1 \%)$ patients, mainly at TBH $(n=15)$. An international normalised ratio (INR) was performed on $8(2.6 \%)$ patients; mainly at GSH $(n=4)$ and TBH $(n=3)$. Cholesterol levels were checked in $5(1.6 \%)$ patients, all of whom were investigated at GSH. C-reactive protein (CRP) levels were measured in $4(1.3 \%)$ patients at Paarl Hospital.

\section{Discussion}

Previous studies had shown a male predominance in patients presenting with first-onset seizures ${ }^{[6,8]}$ Our study, however, showed the exact opposite, with $162(61.2 \%)$ female patients. The age of patients in this study was similar in range to previously studied groups: the most common age of presentation was between 31 and 40 years. ${ }^{[0,8]}$

Only $71 \%$ had neuroimaging performed as part of their diagnostic work-up. No MRIs were performed in this study - reflecting the limited access to this investigation (MRIs being available only at tertiary hospitals and usually during office hours, except in emergencies). It may be speculated that doctors working in ECs were seeking to limit costs on those patients who had recovered completely from their seizure by not ordering CT scans for them. It is also possible that to facilitate patient flow, patients were discharged before CT scanning.

The largest proportion of urgent CT scans (66\%) was performed at the two tertiary centres where it may be thought to be more convenient to the patient, diminishing also the need for further appointments and follow-up. Since these hospitals are the referral centres for all the secondary level (and some primary level) facilities in the Western Cape, it is possible that patients were referred solely for such investigations. An alarming trend was noticed in a large percentage of patients (85\%) - they did not undergo the elective CT scan booked for them. The main reasons were: patients not attending their scheduled appointments, dates not communicated to patients, and patients being discharged prior to appointments being made. Taking the above in consideration, it may be better to complete investigations on an emergent basis in the EC to expedite patient treatment and disposal.

There is some debate in the literature regarding the appropriate timing of neuroimaging. The US guideline states that imaging could be deferred to an elective date, provided there is adequate patient follow-up ${ }^{[2]}$ The UK guidelines are similar, requiring imaging within 4 weeks. ${ }^{[3]}$ Acknowledging that keeping patients in the EC contributes to overcrowding, in the SA setting it may be more appropriate to obtain CT scans emergently (within 24 hours). It is important to note that the debate regarding timing of imaging is only applicable to patients who have returned to a normal neurological baseline and have no significant co-morbidities. Any clinical suspicion of intracranial pathology demands emergent imaging. ${ }^{[11]}$

The yield of abnormal scans (59.6\%) was higher than in international studies. ${ }^{[5,6]}$ Unfortunately, data regarding abnormal findings 


\section{RESEARCH}

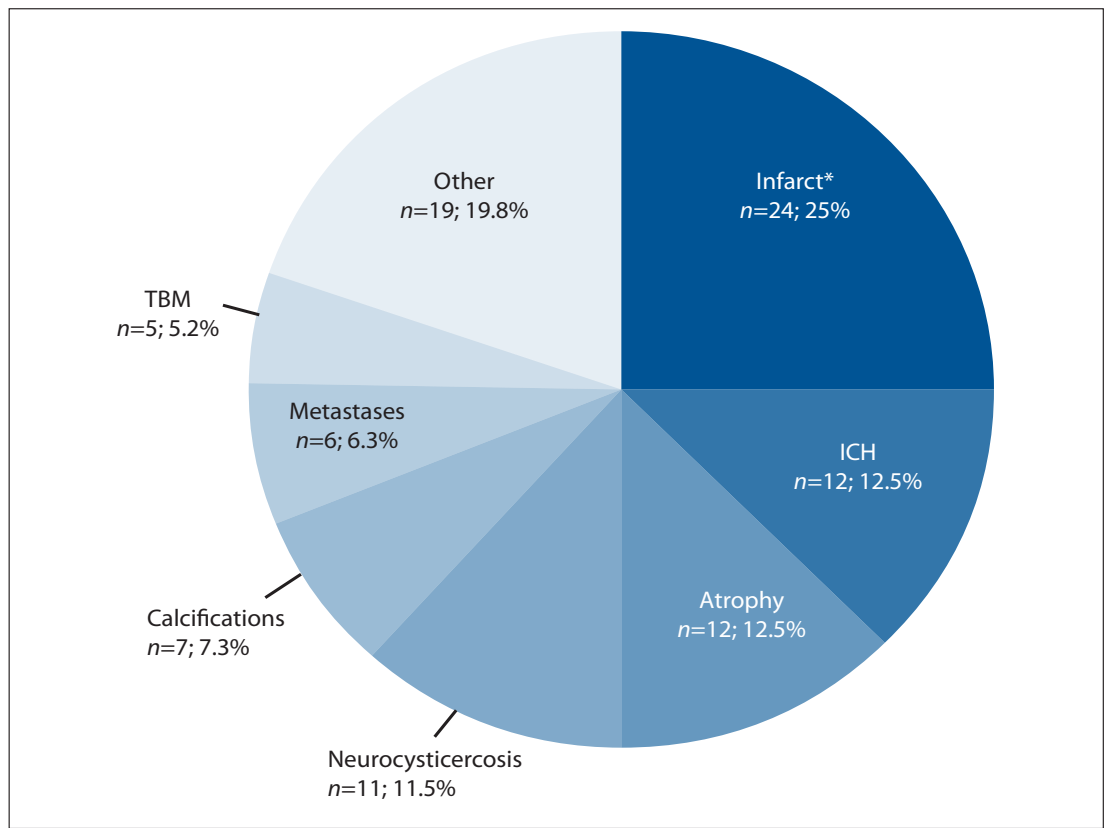

Fig. 1. Abnormalities on computed tomography scans of the brain deemed causative for seizure activity (ICH = intracranial haemorrhage; TBM = tuberculous meningitis; ${ }^{*}$ old and new infarcts).

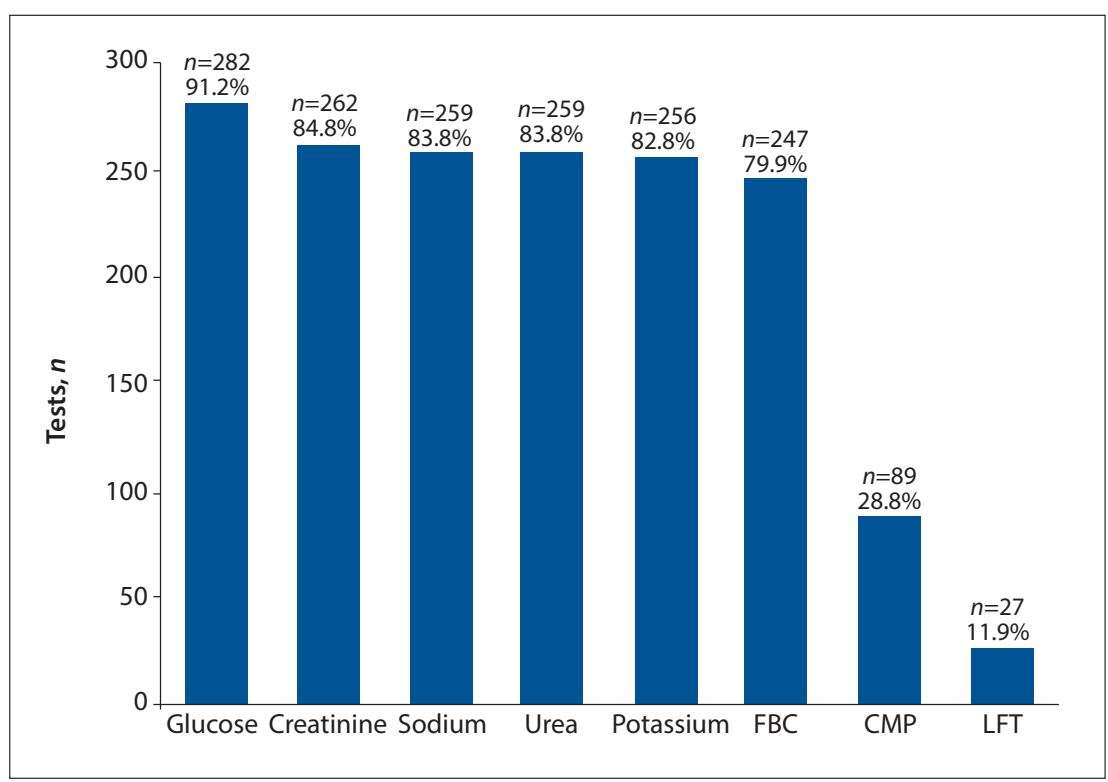

Fig. 2. Most common biochemical investigations performed in patients presenting with first-onset seizures $(F B C=$ full blood count; $C M P=$ calcium-magnesium-phosphate; $L F T=$ liver functions test $)$.

on clinical neurological examinations were not collected, but it is possible that the higher positive yield was a reflection of the high number of HIV-positive patients in the Western Cape (particularly considering the high prevalence of neurocysticercosis and tuberculous meningitis (TBM)). ${ }^{[15]}$ Atrophy, traumatic brain injury and tumours were the most common CT scan abnormalities in international studies, different from the ranking order of our findings. International data show an increased prevalence in those over 60 years of age, which was not a finding in our study (Table 1). ${ }^{[1]}$
Themostcommonbiochemicalabnormalities causing seizures are related to serum glucose and sodium. ${ }^{[5,7]}$ Most international guidelines suggest that plasma electrolytes, glucose and calcium be measured, if indicated. Yet $>80 \%$ of patients in our study received a full renal and electrolyte screen as well as a complete haematological investigation. While it is difficult to comment on whether these investigations were unnecessary (as the patients' end diagnoses were not correlated to the investigations performed), what is more concerning is that essential blood tests, such as glucose, were not undertaken in all patients. The high yield of blood investigations performed at tertiary hospitals may be explained by the greater number of internal medicine specialists in their ECs and the fact that patients investigated there are likely to have had more complicated medical diagnoses and co-morbidities. The abnormal yield of the blood tests was not reviewed. Further analyses might assist in guiding a rational choice of blood investigations, particularly to limit costs. The inclusion of certain tests are a reflection of the current practice at particular centres: syphilis testing at TBH is a good example. The introduction of a guideline would assist doctors working in ECs to make better evidence-based decisions.

There is no evidence of occult bacterial meningitis presenting as isolated seizures and it can be argued that an LP is indicated only in patients who have meningitis clinically or other systemic signs suggesting an intracranial process such as vasculitis, provided that there is no suspicion of raised intracranial pressure. Adherence to this advice may account for the small number of patients (22\%) in whom an LP was performed. Since HIVpositive patients presenting with seizures, have a high incidence of cryptococcal meningitis and TBM there should be a low threshold for LP provided that there are no contraindications..$^{[13,14]}$

EEG showing presence of focal seizures may assist with localising the site involved and predict seizure recurrence, as well as signalling subclinical status epilepticus. Normal EEG findings do not exclude the diagnosis of epilepsy. The abnormal yield of EEGs in our study (18\%) was well below the $50 \%$ reported in other studies. ${ }^{[4]}$ All eight of the urgent EEGs were performed at tertiary hospitals. There are clear indications for urgent EEG (e.g. overt or subclinical status epilepticus), but since patients' clinical examination was not included in this study, it is difficult to comment on whether these EEGs were appropriate. Recent literature suggests that an EEG should not be part of investigations ordered from the $\mathrm{EC}$, but should be left to the discretion of specialist neurology services. ${ }^{[12]}$ This approach seems reasonable in our setting, but also implies that we are underutilising this resource (especially in the patient group where status epilepticus or non-epileptic psychogenic seizures are suspected).

HIV-positive patients have a higher incidence of neurological disease-causing seizures. ${ }^{[13]}$ Of our study population, $16 \%$ were confirmed HIV-positive, but their HIV status did not appear to influence either their 
Table 3. HIV subgroup analysis in patients presenting with first-onset seizures

\begin{tabular}{|c|c|c|c|}
\hline & \multicolumn{3}{|c|}{$\begin{array}{c}\text { HIV status } \\
n\end{array}$} \\
\hline & Positive & Negative & Unknown \\
\hline Total, $N$ & 49 & 53 & 207 \\
\hline \multicolumn{4}{|l|}{ Seizure type } \\
\hline Focal & 9 & 10 & 22 \\
\hline Generalised & 40 & 43 & 185 \\
\hline \multicolumn{4}{|l|}{ CT scans ordered } \\
\hline Urgent & 37 & 31 & 83 \\
\hline Elective & 4 & 9 & 54 \\
\hline Not ordered & 8 & 13 & 70 \\
\hline $\begin{array}{l}\text { Planned but not } \\
\text { performed }\end{array}$ & 4 & 4 & 41 \\
\hline \multicolumn{4}{|l|}{ CT scan results } \\
\hline Abnormal & 24 & 20 & 56 \\
\hline Normal & 13 & 16 & 40 \\
\hline \multicolumn{4}{|l|}{ Lumbar punctures } \\
\hline Abnormal & 7 & 7 & 3 \\
\hline Normal & 16 & 12 & 24 \\
\hline Not done & 26 & 34 & 180 \\
\hline \multicolumn{4}{|l|}{ EEG } \\
\hline Abnormal & 3 & 1 & 1 \\
\hline Normal & 0 & 12 & 3 \\
\hline Not done & 47 & 50 & 192 \\
\hline
\end{tabular}

diagnostic work-up or the abnormal yield of investigations (Table 3 ). The cause of these contradictory results is unclear, but the high proportion of patients (67\%) with an unknown HIV status might have been a confounding factor. Point-of-care HIV tests are readily available and more emphasis on HIV testing in the SA setting might be warranted.

\section{Study limitations}

Very few cases were identified prospectively by EC staff, forcing the investigators to rely on the EC registers. The record-keeping in some of the EC registers was poor, with missing data. This inadvertently led to some patients being missed. The clinical record-keeping was also below standard and information sometimes impossible to obtain. A small number of patients might have been duplicated in two hospitals' registers, particularly when referred for urgent neuroimaging.

Missing data may have confounded data analysis, suggesting that a greater patient number might have been obtained. This may have affected the internal validity of the study.
Another limitation was that the clinical presentation, neurological examination and test results were not reviewed, nor was the final diagnosis correlated with investigations undertaken. Valuable information regarding the diagnosis and prognosis of patients might have been lost.

Our study was performed on a small population in one province in SA, and is thus potentially not generalisable to other countries or even other provinces.

\section{Conclusion}

The findings of this study emphasise the need for a local guideline regarding the investigation of first-onset seizures in adults. We have demonstrated wide local variance for all types of investigations performed. A local guideline would direct doctors in respect of appropriate investigations, thus ensuring better quality patient care and, potentially, saving costs.

Further study as to the aetiology of first-onset seizures in SA, especially in HIV-positive patients, is needed. The cost implications of inappropriate tests, particularly in SA's resource-limited setting, should be further investigated.

\section{References}

1. Hauser W, Behgi E. First seizure definitions and worldwide incidence and mortality. Epilepsia 2008:49(Suppl 1):8-12. [http://dx.doi.org/10.1111/j.1528-1167.2008.01443.x]

2. ACEP Clinical Policies Committee, Clinical Policies Subcommittee on Seizures. Clinical policy: Critical issues in the evaluation and management of adult patients presenting to the emergency department issues in the evaluation and management of adult patients presenting to the emergency department
with seizures. Ann Emerg Med 2004;43(5):605-625. [http://dx.doi.org/10.1016/S019606440400068X] 3. National Institute for Health and Clinical Excellence. The Epilepsies: The Diagnosis and Management 3. National Institute for Health and Clinical Excellence. The Epilepsies: The Diagnosis and Management
of the Epilepsies in Adults and Children in Primary and Secondary Care. Manchester: NICE, 2012. http://www.nice.org.uk/nicemedia/live/13635/57779/57779.pdf (accessed 27 October 2012).

4. King M, Newton M, Jackson G, et al. Epileptology of the first-seizure presentation: A clinical, electroencephalographic and magnetic resonance imaging study of 300 consecutive patients. Lancet 1998;352(9133):1007-1010. [http://dx.doi.org/10.1016/S0140-6736(98)03543-0]

5. Tardy B, Lafond P, Convers P, et al. Adult first generalized seizure: Etiology, biological tests, EEG, CT scan, in an ED. Am J Emerg Med 1995;13(1):1-5. [http://dx.doi.org/10.1016/0735-6757(95)90229-5]

6. Breen D, Dunn M, Davenport A, et al. Epidemiology, clinical characteristics and management of adults referred to a teaching hospital first seizure clinic. Postgrad Med J 2005;81(961):715-718. [http://dx.doi. org/10.1136/pgmj.2004.031203]

7. Turnbull TL, Vanden Hoek TL, Howes DS, et al. Utility of laboratory studies in the emergency department patient with a new-onset seizure. Ann Emerg Med 1990;19(4):373-377. [http://dx.doi. copartment patient with a new-onset

8. Henneman P. DeRoos F, Lewis R. Determining the need for admission in patients with new-onset Henneman P, DeRoos F, Lewis R. Determining the need for admission in patients with new-onset
seizures. Ann Emerg Med 1993;24(6):1108-1114. [http://dx.doi.org/10.1016//0196-0644(94)70240-3] 9. Pesola G, Westfal R. New-onset generalised seizures in patients with AIDS presenting to an emergency department. Acad Emerg Med 1998;5(9):905-911. [http://dx.doi.org/10.1111/j.1553-2712.1998. tb02820.x]

10. Sempere A, Villaverde F, Martinez-Menedez B, et al. First seizure in adults: A prospective study from the emergency department. Acta Neurol Scand 1992;86(2):134-138. [http://dx.doi. org/10.1111/j.1600-0404.1992.tb05054.x]

11. Krumholz A, Wiebe S, Gronseth G, et al. Practice parameter: Evaluating an apparent unprovoked first seizure in adults (an evidence based review). Report of the Quality Standards Subcommittee of the American Academy of Neurology and the American Epilepsy Society. Neurology 2007;69(21):19962007. [http://dx.doi.org/10.1212/01.wnl.0000285084.93652.43]

12. Dunn M, Breen D, Davenport R, et al. Early management of adults with an uncomplicated first generalised seizure. Emerg Med J 2005;22(4):237-242. [http://dx.doi.org/10.1136/emj.2004.015651]

3. Kellinghaus C, Engbring C, Kovac, et al. Frequency of seizures and epilepsy in neurological HIV. Kellinghaus C, Engbring C, Kovac, et al. Frequency of seizures and epilepsy in neurological
infected patients. Seizure 2008;17(1):27-33. [http://dx.doi.org/10.1016/.seizure.2007.05.017]

infected patients. Seizure 2008;17(1):27-33. [http://dx.doi.org/10.1016/j.seizure.2007.05.017]
14. Modi G, Modi M, Martinus I, et al. New onset seizures associated with HIV infection. Neurology 2000;55(5):1558-1560. [http://dx.doi.org/10.1111/j.1528-1167.2008.01942.x]

15. Statistics South Africa. Mid year population estimates, 2010. Pretoria: StatsSA, 2012. http://www. statssa.gov.za/publications/P0302/P03022010.pdf (accessed October 2012).

Accepted 7 August 2013 